\title{
CONTINUITY OF OPERATORS INTERTWINING WITH TRANSLATION OPERATORS ON HYPERGROUPS
}

\author{
VISHVESH KUMAR AND RITUMONI SARMA
}

\begin{abstract}
Let $K$ be a commutative or compact hypergroup. Let $\mu$ be a bounded complex-valued Borel measure on $K$, and let $T_{\mu}$ be the corresponding convolution operator of $L^{1}(K)$. Let $S$ be a bounded linear operator on a Banach space $X$. We prove that every linear operator $\Psi: X \rightarrow L^{1}(K)$ such that $\Psi S=T_{\mu} \Psi$ is continuous if and only if the pair $\left(S, T_{\mu}\right)$ has no critical eigenvalues.
\end{abstract}

\section{INTRODUCTION}

A hypergroup is a locally compact Hausdorff space equipped with a convolution product which maps two points to a probability measure with compact support. Hypergroups are probabilistic generalizations of locally compact groups where the convolution of two points is a point mass measure. The study of hypergroups began in the 1970s with the works of Dunkl [4], Spector [15] and Jewett [6]. Hypergroups are referred to as convos by Jewett. The class of translation invariant operators is the fundamental object in harmonic analysis. Several authors studied translation invariant operators on hypergroups $[13,8,9,12,11]$.

Laursen and Neumann asked [10, Open Problem 6.3.3] the following question which is essentially a generalization of a question of Johnson [7, Note 8(iii)] on the continuity of the intertwiner on $L^{1}(\mathbb{R})$.

"Let $\mu$ and $\nu$ be complex valued bounded Borel measures on a locally compact abelian group $G$ such that the corresponding pair $\left(T_{\mu}, T_{\nu}\right)$ of convolution operators on $L^{1}(G)$ has no critical eigenvalues. Is every linear operator $\Psi: L^{1}(G) \rightarrow L^{1}(G)$ for which $T_{\mu} \Psi=\Psi T_{\nu}$, continuous?"

The above question was solved by C. Aparicio and A. R. Villena [2] in 2002. In 2008, J. Alaminos et. al. [1] extended this result to the MAP class groups. The aim of this article is to extend the question of Johnson to the locally compact abelian hypergroups

2010 Mathematics Subject Classification. Primary 43A62, 43A22; Secondary 46H10.

Key words and phrases. hypergroup, multipliers, automatic continuity, translation invariant operators. 
and compact hypergroups (possibly non-abelian). For the proof of our results we adopt the techniques presented in [2].

Section 2 contains the basics of hypergroups in the form we need. In Section 3, we state and prove the main results, namely, Theorem 3.2 and Theorem 3.3 in the context of commutative hypergroups and compact hypergroups respectively.

\section{Preliminaries}

Let $K$ be a locally compact hypergroup and let $M(K)$ be the space of all bounded Borel complex-valued measures. We mainly follow Jewett [6] for basic notation and concepts. We denote the involution of any element $x$ of $K$ by $\check{x}$, which is denoted by $x^{-}$in [6]. The hypergroup algebra $L^{1}(K)$ is a Banach *-algebra. $L^{1}(K)$ is a closed two sided ideal of $M(K)$. For $\mu \in M(K)$, the convolution operator $T_{\mu}: L^{1}(K) \rightarrow L^{1}(K)$ is defined as $T_{\mu}(f)=\mu * f$. Denote the space of bounded continuous complex valued functions on $K$ by $C^{b}(K)$. Now, assume that $K$ is a commutative hypergroup. Let

$$
\mathcal{X}_{b}(K)=\left\{\chi \in C^{b}(K): \chi(e)=1 \text { and } \chi(x * y)=\chi(x) \chi(y) \forall x, y \in K\right\}
$$

Equip $\mathcal{X}_{b}(K)$ with the compact-open topology so that it is a locally compact Hausdorff space. By [3, Theorem 2.2.2], the structure space of $L^{1}(K)$ can be identified with $\mathcal{X}_{b}(K)$. Let

$$
\widehat{K}=\left\{\chi \in \mathcal{X}_{b}(K): \chi(\check{x})=\overline{\chi(x)} \forall x \in K\right\}
$$

The set $\widehat{K}$ is also equipped with the compact-open topology. As in [3, Example 2.2.49] we see that $\mathcal{X}_{b}(K)$ may not be equal to $\widehat{K}$. Note that these spaces need not possess a hypergroup structure. See [6, Example $9.3 \mathrm{C}]$.

The Fourier transform of $f \in L^{1}(K)$ is defined by

$$
\hat{f}(\chi)=\int_{K} f(x) \overline{\chi(x)} d m(x) \forall \chi \in \widehat{K} .
$$

Similarly, the Fourier-Stieltjes transform of $\mu \in M(K)$ is defined by

$$
\hat{\mu}(\chi)=\int_{K} \overline{\chi(x)} d \mu(x) \forall \chi \in \widehat{K} .
$$

By [3, Theorem 2.2.4], the mapping $\mu \mapsto \hat{\mu}$ is a norm decreasing *-algebra homomorphism from $M(K)$ to $C^{b}(\widehat{K})$. Further, $\hat{f}$ vanishes at infinity. 
In the remaining part of this section, $K$ denotes a compact hypergroup. The Haar measure $m$ on $K$ is chosen so that $m(K)=1$. An irreducible representation $\pi$ of $K$ is an irreducible $*$ - algebra representation of $M(K)$ into $\mathcal{L}\left(\mathcal{H}_{\pi}\right)$ for some Hilbert space $\mathcal{H}_{\pi}$ such that

(i) $\pi(e)=I$ and

(ii) for each $u, v \in \mathcal{H}_{\pi}$, the mapping $\mu \mapsto\langle\pi(\mu) u, v\rangle$ is continuous from $M(K)^{+}$to $\mathbb{C}$, where $M(K)^{+}$is equipped with the weak (cone) topology.

Vrem [16] proved that every irreducible representation of a compact hypergroup is finite dimensional. We denote by $\widehat{K}$ the set of all equivalence classes of irreducible representations of $K$. For any $\pi \in \widehat{K}$, let $\bar{\pi}$ denote the conjugate representation. It is wellknown that irreducible representations of $K$ separate the points of $L^{1}(K)$. Indeed, for any $f, g \in L^{1}(K)$, suppose $\pi(f)=\pi(g)$ for all $\pi \in \widehat{K}$, so that $\hat{f}(\bar{\pi})=\hat{g}(\bar{\pi}) \forall \pi \in \widehat{K}$, i.e., $(f-g \hat{)}(\bar{\pi})=0 \forall \pi \in \widehat{K}$. Now, by [16, Theorem 3.3], $f \mapsto \hat{f}$ is a norm decreasing *algebra homomorphism from $L^{1}(K)$ to $\mathcal{E}_{0}(K)$ (for notation, see [5, Definition 28.24]). So we get $\|f-g\| \leq\|(f-g)\|_{\mathcal{E}_{0}(K)}=0$, which implies that $f=g$. Hence $\widehat{K}$ separates the points of $L^{1}(K)$. For more details one can refer to [16] and [3].

\section{Operators COMmuting With CONVOLUtion OPERATORS}

Suppose $X$ and $Y$ are Banach spaces. Let $S$ and $T$ be bounded linear operators on $X$ and $Y$ respectively. A linear operator $\Psi: X \rightarrow Y$ is said to intertwine the pair $(S, T)$ if $\Psi S=T \Psi$. A complex number $\lambda$ is called a critical eigenvalue of $(S, T)$ if $\lambda$ is an eigenvalue of $T$ and $\left(\lambda I_{X}-S\right)(X)$ is a subspace of infinite codimension in $X$. It is known that if $(S, T)$ has a critical eigenvalue then there exists a discontinuous operator which intertwines the pair $(S, T)$ (see [14, Lemma 3.2]).

For a linear operator $\Psi$ from $X$ to $Y$, the following subspace of $Y$ is called the separating space of $\Psi$ :

$$
\mathfrak{S}(\Psi):=\left\{y \in Y: \text { there exists }\left(x_{n}\right) \rightarrow 0 \text { in } X \text { with } \Psi\left(x_{n}\right) \rightarrow y\right\}
$$

The separating space of an operator plays an important role in the study of continuity of linear operators in automatic continuity theory. It is an easy consequence of the closed 
graph theorem that an operator $\Psi$ is continuous if and only if $\mathfrak{S}(\Psi)=0$. For more details see $[14]$.

Lemma 3.1. [14, Lemma 1.6 (Stability Lemma)] Let $X$ and $Y$ be Banach spaces and let $\left(T_{n}\right)$ and $\left(R_{n}\right)$ be sequences of continuous operators on $X$ and $Y$, respectively. If $S$ is a linear operator from $X$ into $Y$ satisfying $S T_{n}=R_{n} S$ for all $n \in \mathbb{N}$, then there is an integer $N$ such that

$$
\overline{\left(R_{1} R_{2} \cdots R_{n} \mathfrak{S}\right)}=\overline{\left(R_{1} R_{2} \cdots R_{N} \mathfrak{S}\right)} \quad \text { for all } n \geq N
$$

In the main result of this article, we deal with the above mentioned question of Johnson in the context of hypergroup algebras. In fact, we show that the answer to his question is affirmative when the hypergroup is commutative or compact. In the general case, even when the hypergroup is a locally compact group which is not one of the MAP class groups, the answer to this question is not known.

Theorem 3.2. Let $K$ be a commutative hypergroup with its dual $\widehat{K}$ and let $T_{\mu}$ be the convolution operator corresponding to $\mu \in M(K)$ on $L^{1}(K)$. Suppose $S$ is a linear operator on a Banach space $X$. Suppose the pair $\left(S, T_{\mu}\right)$ has no critical eigenvalues. Then every linear operator $\Psi: X \rightarrow L^{1}(K)$ which intertwines $\left(S, T_{\mu}\right)$ is continuous.

Proof. We will prove this theorem by contradiction. Suppose that $\Psi$ is a discontinuous operator and hence the separating space $\mathfrak{S}(\Psi) \neq 0$.

We claim that the set

$$
\{\hat{\mu}(\chi): \chi \in \widehat{K} \text { and } \hat{f}(\chi) \neq 0 \text { for some } f \in \mathfrak{S}(\Psi)\}
$$

is a finite set. Suppose that it is infinite. Let $\left(\chi_{n}\right)$ be a sequence in $\widehat{K}$ such that $\hat{\mu}\left(\chi_{m}\right) \neq$ $\hat{\mu}\left(\chi_{n}\right)$ for $m \neq n$ and for every $n \in \mathbb{N}$ there exists a $f_{n} \in \mathfrak{S}(\Psi)$ such that $\hat{f}_{n}\left(\chi_{n}\right) \neq 0$. For our convenience we will denote $\hat{\mu}\left(\chi_{n}\right)$ by $\lambda_{n}$.

Since $\Psi$ intertwines the pair $\left(S, T_{\mu}\right)$, we get $\left(\lambda_{n} I_{K}-T_{\mu}\right) \Psi=\Psi\left(\lambda_{n} I_{X}-S\right)$ for each $n \in \mathbb{N}$ where $I_{K}$ and $I_{X}$ denote the identity operator on $L^{1}(K)$ and $X$ respectively. Then by Lemma 3.1, it follows that there exists an $n \in \mathbb{N}$ such that

$$
\overline{\left(\lambda_{1} I_{K}-T_{\mu}\right) \cdots\left(\lambda_{n} I_{K}-T_{\mu}\right)(\mathfrak{S}(\Psi))}=\overline{\left(\lambda_{1} I_{K}-T_{\mu}\right) \cdots\left(\lambda_{n+1} I_{K}-T_{\mu}\right)(\mathfrak{S}(\Psi))}
$$


where $\bar{A}$ denotes the topological closure of the set $A$. It is easy to see that the Fourier transform of every function of the hindmost space vanishes at $\chi_{n+1}$. Indeed, we have for each $f \in L^{1}(K)$

$\left[\left(\lambda_{1} I_{K}-T_{\mu}\right) \cdots\left(\lambda_{n+1} I_{K}-T_{\mu}\right)(f)\right]^{\wedge}\left(\chi_{n+1}\right)=\left(\lambda_{1}-\hat{\mu}\left(\chi_{n+1}\right)\right) \cdots\left(\lambda_{n+1}-\hat{\mu}\left(\chi_{n+1}\right)\right) \hat{f}\left(\chi_{n+1}\right)=0$.

Now, by the continuity of the Fourier transform at $\chi_{n+1}$ we covet the conclusion.

In particular, the Fourier transform of every function of the set $\left(\lambda_{1} I_{K}-T_{\mu}\right) \cdots\left(\lambda_{n} I_{K}-\right.$ $\left.T_{\mu}\right)(\mathfrak{S}(\Psi))$ vanishes at $\chi_{n+1}$. Therefore,

$$
\left(\lambda_{1}-\lambda_{n+1}\right) \cdots\left(\lambda_{n}-\lambda_{n+1}\right) \hat{f}\left(\chi_{n+1}\right)=\left[\left(\lambda_{1} I_{K}-T_{\mu}\right) \cdots\left(\lambda_{n} I_{K}-T_{\mu}\right)(f)\right]^{\wedge}\left(\chi_{n+1}\right)=0
$$

for each $f \in \mathfrak{S}(\Psi)$. Therefore, $\left(\lambda_{1}-\lambda_{n+1}\right) \cdots\left(\lambda_{n}-\lambda_{n+1}\right) \hat{f}_{n+1}\left(\chi_{n+1}\right)=0$. But $\left(\lambda_{1}-\lambda_{n+1}\right)$. $\cdots\left(\lambda_{n}-\lambda_{n+1}\right) \neq 0$, so $\hat{f}_{n+1}\left(\chi_{n+1}\right)=0$, which contradicts the choice of $f_{n+1}$.

Hence, there exist $\beta_{1}, \beta_{2}, \cdots \beta_{m} \in \mathbb{C}$ such that

$$
\{\hat{\mu}(\chi): \chi \in \widehat{K} \text { and } \hat{f}(\chi) \neq 0 \text { for some } f \in \mathfrak{S}(\Psi)\}=\left\{\beta_{1}, \beta_{2}, \cdots \beta_{m}\right\}
$$

This shows that $\left(\beta_{1} I_{K}-T_{\mu}\right) \cdots\left(\beta_{m} I_{K}-T_{\mu}\right)(\mathfrak{S}(\Psi))=0$.

Without loss of generality, by removing some of the scalars $\beta_{j}$, we can assume that $\beta_{1}, \beta_{2}, \cdots, \beta_{m}$ are eigenvalues of $T_{\mu}$. On the other hand, since $\left(S, T_{\mu}\right)$ has no critical eigenvalues, one can conclude that for each $j \in\{1,2, \cdots m\}$, the range of $\left(\beta_{j} I_{X}-S\right)$ has finite co-dimension.

Set $N=\left(\beta_{1} I_{X}-S\right)\left(\beta_{2} I_{X}-S\right) \cdots\left(\beta_{m} I_{X}-S\right)(X)$. It follows from the above paragraph that the co-dimension of $N$ is finite. It follows from [14, Lemma 3.3] that $N$ is a closed subspace of $X$. Suppose $R$ denotes the bounded linear operator from $X$ onto $N$ given by $R(x)=$ $\left(\beta_{1} I_{X}-S\right)\left(\beta_{2} I_{X}-S\right) \cdots\left(\beta_{m} I_{X}-S\right)(x)$ for each $x \in X$ and denote the restriction of $\Psi$ to $N$ by $\Phi$. Then $\Phi R=\left(\beta_{1} I_{K}-T_{\mu}\right) \cdots\left(\beta_{m} I_{K}-T_{\mu}\right) \Psi$. Since $\left(\beta_{1} I_{K}-T_{\mu}\right) \cdots\left(\beta_{m} I_{K}-T_{\mu}\right)(\mathfrak{S}(\Psi))=0$, by [14, Lemma 1.3(i)], $\Phi R$ is continuous.

Now, we show that $\Phi$ is continuous. Suppose $\left(y_{n}\right)$ is a sequence $\left(y_{n}\right)$ in $N$ with $\lim y_{n}=0$. By the open mapping theorem, $R$ is open, and hence there exists a sequence $\left(x_{n}\right)$ in $X$ such that $\lim x_{n}=0$ and $R\left(x_{n}\right)=y_{n}$ for each $n \in \mathbb{N}$. Now using the continuity of $\Phi R$, we have $\Phi\left(y_{n}\right)=\lim \Phi R\left(x_{n}\right)=0$ and this shows that $\Phi$ is continuous.

Finally, since $\Psi$ is continuous on a finite co-dimensional subspace $N$, it is continuous and therefore, we have got a contradiction. Hence, the result follows. 
Theorem 3.3. Let $K$ be a compact hypergroup and let $T_{\mu}$ be the convolution operator corresponding to $\mu \in M(K)$. Suppose $S$ is a bounded linear operator on a Banach space $X$. Suppose that $\left(S, T_{\mu}\right)$ has no critical eigenvalues. Then every intertwiner map $\Psi: X \rightarrow$ $L^{1}(K)$ is continuous.

Proof. To prove the result, it is enough to show that the separating space $\mathfrak{S}(\Psi)=\{0\}$. In order to prove this, we show that there exists a non zero polynomial $p$ such that $p\left(T_{\mu}\right)(\mathfrak{S}(\Psi))=0$. Assume the contrary, that is, $p\left(T_{\mu}\right)(\mathfrak{S}(\Psi)) \neq 0$ for each non-zero polynomial $p$. This demands that $\mathfrak{S}(\Psi) \neq 0$. Since $\widehat{K}$ separates points of $L^{1}(K)$, there exists a $\rho_{1} \in \widehat{K}$ such that $\pi_{1}(\mathfrak{S}(\Psi)) \neq 0$. Let $p_{1}$ be the characteristic polynomial of $\pi_{1}(\mu)$. We can choose inductively $\rho_{n+1} \in \widehat{K}$ such that $\rho_{n+1}\left(\left(p_{1} \cdots p_{n}\right)\left(T_{\mu}\right)(\mathfrak{S}(\Psi)) \neq 0\right.$ for each $n \in \mathbb{N}$, where $p_{i}$ is the characteristic polynomial of $\rho_{i}(\mu)$ for $i \in\{1,2, \cdots, n\}$.

Since $\Psi$ intertwines $S$ and $T_{\mu}$, we have $p_{n}(S) \Psi=\Psi p_{n}\left(T_{\mu}\right)$ for each $n \in \mathbb{N}$. By Lemma 3.1 , there exists $m \in \mathbb{N}$ such that

$$
\overline{\left(p_{1} \cdots p_{m}\right)\left(T_{\mu}\right)(\mathfrak{S}(\Psi))}=\overline{\left(p_{1} \cdots p_{m+1}\right)\left(T_{\mu}\right)(\mathfrak{S}(\Psi))} .
$$

Observe that if $f \in\left(p_{1} \cdots p_{n}\right)\left(T_{\mu}\right)(\mathfrak{S}(\Psi))$ then $\pi_{m+1}(f)=0$. Indeed, $f=\left(p_{1} \cdots p_{n}\right)\left(T_{\mu}\right)(g)$ for some $g \in \mathfrak{S}(\Psi)$ and

$$
\pi_{m+1}(f)=\pi_{m+1}\left(\left(p_{1} \cdots p_{n}\right)\left(T_{\mu}\right)(g)\right)=p_{1}\left(\pi_{m+1}(\mu)\right) \cdots p_{m+1}\left(\pi_{m+1}(\mu)\right) \pi_{m+1}(g)=0
$$

as $p_{m+1}$ is the characteristic polynomial of $\pi_{m+1}(\mu)$ so it annihilates $\pi_{m+1}(\mu)$. Therefore,

$$
\pi_{m+1}\left(\left(p_{1} \cdots p_{m}\right)\left(T_{\mu}\right)(\mathfrak{S}(\Psi))\right)=\{0\}
$$

which contradicts the choice of $\pi_{m+1}$. Therefore, $p\left(T_{\mu}\right)(\mathfrak{S}(\Psi))=\{0\}$ for some non zero polynomial $p$. Hence, there exist $\beta_{1}, \beta_{2}, \cdots, \beta_{n} \in \mathbb{C}$ such that

$$
\left(\beta_{1} I_{K}-T_{\mu}\right) \cdots\left(\beta_{k} I_{K}-T_{\mu}\right) \mathfrak{S}(\Psi)=\{0\}
$$

where $I_{K}$ is the identity operator on $L^{1}(K)$. Now, the rest of the proof follows from the proof of Theorem 3.2 . 


\section{ACKNOWLEDGMENT}

The authors thank the reviewer for his/her valuable comments/suggestions. Vishvesh Kumar is supported by FWO Odysseus 1 grant G.0H94.18N: Analysis and Partial Differential Equations.

\section{REFERENCES}

[1] J. Alaminos, J. Extremera and A. R. Villena, A note on the continuity of operators intertwining with convolution operators, Taiwanese J. Math. 12(2) (2008) 337-340.

[2] C. Aparicio and A. R. Villena, Continuity of operators intertwining with convolution operators, J. Funct. Anal. 196 (2002) 155-161.

[3] W. R. Bloom and Herbert Heyer, Harmonic analysis on probability measures on hypergroups, De Gruyter, Berlin (1995).

[4] C. F. Dunkl, The measure algebra of a locally compact hypergroup, Trans. Amer. Math. Soc 179 (1973) 331-348.

[5] Hewitt, Edwin and K. A. Ross, Abstract harmonic analysis. Vol. II: Structure and analysis for compact groups. Analysis on locally compact Abelian groups, Die Grundlehren der mathematischen Wissenschaften, Band 152 Springer-Verlag, New York-Berlin (1970).

[6] R. I. Jewett, Space with an abstract convolution of measures, Adv. in math, 18 (1975) $1-101$.

[7] B. E. Johnson, Continuity of linear operator commuting with continuous linear operators, Trans. Amer. Math, Soc. 156 (1967) 88-102.

[8] V. Kumar, R. Sarma, and N. Shravan Kumar, Orlicz spaces on hypergroups. Publ. Math. Debrecen 94 (2019), no. 1-2, 31-47.

[9] Vishvesh Kumar, N. Shravan Kumar and R. Sarma, Unbounded translation invariant operators on commutative hypergroups. Methods Funct. Anal. Topology 25 (2019), no. 3, 236-247.

[10] K. B. Laursen and M. M. Neumann, An introduction to local spectral theory, London Mathematical Soc. Monograph, New Series, Vol. 20. The Clarendon Press, Oxford Univ. Press, New York, (2000).

[11] Pavel, L. Multipliers for the Lp-spaces of a hypergroup. Rocky Mountain J. Math. 37 (2007), no. 3, 987-1000.

[12] L. Szeḱelyhidi, S. M. Tabatabaie, and B. H. Sadathoseyni, Convolution operators on measure algebras of KPC-hypergroups. Adv. Pure Appl. Math. 10 (2019), no. 1, 1-6.

[13] R. Sarma, N. Shravan Kumar and V. Kumar, Multipliers on vector-valued $L^{1}$-spaces for hypergroups. Acta Math. Sin. (Engl. Ser.) 34 (2018), no. 7, 1059-1073. 
[14] A. M. Sinclair, Automatic continuity of linear operators, Cambridge Univ. Press, Cambridge, UK (1976).

[15] R. Spector, Apercu de la théorie des hypergroupes Lecture Notes in Math. in: Lecture Notes in Math., vol. 497, Springer, Berlin, 1975, pp. 643-673.

[16] R. C. Vrem, Harmonic analysis on compact hypergroups, Pacific J. Math., 85 (1979) 239-251.

VISHVESH KUMAR

Department of Mathematics: Analysis, Logic and Discrete Mathematics

Ghent University, Ghent, 9000 Belgium

Email address: vishveshmishra@gmail.com

Ritumoni SARMa

Department of Mathematics

Indian Institute of Technology Delhi

DELHI - 110 016, INDIA.

Email address: ritumoni@maths.iitd.ac.in 